\title{
Feasibility Study of Early Blood Pressure Management in Extremely Preterm Infants
}

\author{
Beau J. Batton, MD', Lei Li, PhD², Nancy S. Newman, RN ${ }^{1}$, Abhik Das, $\mathrm{PhD}^{3}$, Kristi L. \\ Watterberg, MD ${ }^{4}$, Bradley A. Yoder, MD ${ }^{5}$, Roger G. Faix, MD ${ }^{5}$, Matthew M. Laughon, MD, \\ $\mathrm{MPH}^{6}$, Krisa P. Van Meurs, MD ${ }^{7}$, Waldemar A. Carlo, $\mathbf{M D}^{8}$, Rosemary D. Higgins, $\mathbf{M D}^{9}$, and \\ Michele C. Walsh, MD, MS ${ }^{1}$ for the Eunice Kennedy ShriverNational Institute of Child \\ Health and Human Development Neonatal Research Network \\ ${ }^{1}$ Department of Pediatrics, Rainbow Babies \& Children's Hospital, Case Western Reserve \\ University, Cleveland, $\mathrm{OH}$ \\ ${ }^{2}$ Statistics and Epidemiology Unit, RTI International, Research Triangle Park, NC \\ ${ }^{3}$ Statistics and Epidemiology Unit, RTI International, Rockville, MD \\ ${ }^{4}$ University of New Mexico Health Sciences Center, Albuquerque, NM \\ ${ }^{5}$ Department of Pediatrics, Division of Neonatology, University of Utah School of Medicine, Salt \\ Lake City, UT \\ ${ }^{6}$ Department of Pediatrics, the University of North Carolina at Chapel Hill, Chapel Hill, NC \\ ${ }^{7}$ Department of Pediatrics, Stanford University School of Medicine, Palo Alto, CA \\ ${ }^{8}$ Division of Neonatology, University of Alabama at Birmingham, Birmingham, AL \\ ${ }^{9}$ Eunice Kennedy Shriver National Institute of Child Health and Human Development, National \\ Institutes of Health, Bethesda, MD
}

\begin{abstract}
Objective-To assess the feasibility of a randomized placebo controlled trial (RCT) of blood pressure (BP) management for extremely preterm infants.

Study design-This was a prospective pilot RCT of infants $23^{0 / 7}-26^{6 / 7}$ weeks gestation who had protocol-defined low BP in the first 24 postnatal hours. Enrolled infants were administered a study infusion (dopamine or placebo) and a study syringe medication (hydrocortisone or placebo).
\end{abstract}

(C) 2012 Mosby, Inc. All rights reserved.

Contact Information for corresponding author Beau Batton, MD, Southern Illinois University School of Medicine (current affiliation), Department of Pediatrics, Division of Neonatology, P.O. Box 19676, Springfield, Illinois 62794, Phone: 217-757-6428, Fax: 217-757-6844, bbatton@ siumed.edu.

List of members of Eunice Kennedy Shriver National Institute of Child Health and Human Development Neonatal Research Network is available at www.jpeds.com (Appendix).

The authors declare no conflicts of interest.

The National Institutes of Health and the Eunice Kennedy Shriver National Institute of Child Health and Human Development (NICHD) provided grant support, including funding from the Best Pharmaceuticals for Children Act, for the Neonatal Research Network's Early Blood Pressure Pilot Study. Data collected at participating sites of the NICHD Neonatal Research Network were transmitted to RTI International, the data coordinating center (DCC) for the network, which stored, managed and analyzed the data for this study.

Publisher's Disclaimer: This is a PDF file of an unedited manuscript that has been accepted for publication. As a service to our customers we are providing this early version of the manuscript. The manuscript will undergo copyediting, typesetting, and review of the resulting proof before it is published in its final citable form. Please note that during the production process errors may be discovered which could affect the content, and all legal disclaimers that apply to the journal pertain. 
Results-Of the 366 infants screened, 119 (33\%) had low BP, 58 (16\%) met all entry criteria, and $10(3 \%)$ were enrolled. 161 (44\%) infants were ineligible because they received early indomethacin. Only $17 \%$ of eligible infants were enrolled. Problems with consent included insufficient time, parent unavailability, and physician unwillingness to enroll critically ill infants. Two infants were withdrawn from the study due to the potential risk of intestinal perforation with simultaneous administration of hydrocortisone and indomethacin.

Conclusions-This pilot RCT was not feasible due to low eligibility and consent rates. An RCT of BP management for extremely preterm infants may require a waiver of consent for research in emergency care. The frequent use of early indomethacin and the associated risk of intestinal perforation when used with hydrocortisone may limit future investigations to only inotropic medications.

\section{Keywords}

Extremely preterm infant; hypotension; hydrocortisone; dopamine; informed consent

The critical condition of most extremely preterm infants in the first week makes identification of a stable population on which to base "normal" blood pressure (BP) data difficult. ${ }^{1-4}$ Uncertainty regarding the acceptable range of $\mathrm{BP}$ also makes deciding when to institute therapy for low $\mathrm{BP}$ challenging and contributes to the wide variation in $\mathrm{BP}$ management for preterm infants. ${ }^{5-9}$ Antihypotensive therapies may raise $\mathrm{BP},{ }^{10,11}$ but there is little evidence to suggest infants benefit from them ${ }^{4,6,12-16}$ and they may all be harmful. ${ }^{9,16-24}$ Compared with untreated infants of a similar gestational age (GA), preterm infants given an antihypotensive therapy have higher rates of death, ${ }^{12,24-27}$ cognitive impairment, ${ }^{26,27}$ deafness, ${ }^{25}$ and cerebral palsy. ${ }^{26}$ These poor outcomes may be due to the etiology of low BP, associated organ hypoperfusion, the treatment of low BP, or a combination of these and other factors.

There are inherent challenges with studying urgent therapeutic interventions shortly after birth in this highly vulnerable patient population. First, the consent process is difficult. An antenatal approach may result in a high number of mothers approached for every infant enrolled ${ }^{28}$ and place an undue burden on women already in a stressful situation. Postnatal consent is difficult by the very short time window available, the high degree of stress parents experience after extremely preterm infant birth, and the frequent administration of medications which affect maternal cognition. Second, clinicians may not have sufficient equipoise to enroll infants in an RCT investigating low BP management because there is not a well established definition of low BP in this population and opinions vary significantly. ${ }^{6-8}$ Lastly, as with any RCT, infants administered study medications may have worse outcomes than those given a placebo because there is a lack of drug safety data within this population.

The lack of large randomized placebo controlled trials (RCT) investigating the use of antihypotensive therapies for preterm infants with low BP in the immediate postnatal period is partially due to the challenges with performing such a trial. This feasibility study of BP management for extremely preterm infants in the immediate postnatal period was performed to assess whether adequate enrollment in an RCT could be achieved using traditional consent mechanisms within a reasonable time frame and without increased risk of morbidity or mortality in any of the study arms.

\section{Methods}

Inborn infants born $23^{0 / 7}-26^{6 / 7}$ weeks GA at one of seven collaborating Eunice Kennedy Shriver National Institute of Child Health and Human Development Neonatal Research Network (NRN) centers were eligible for this study if they had protocol-defined low BP. 
Exclusion criteria included a major birth defect, a decision to withhold or withdraw care, absence of an umbilical arterial catheter (UAC), or recipt of $>20 \mathrm{ml} / \mathrm{kg}$ of fluid boluses, indomethacin, or ibuprofen prior to enrollment. Informed parental consent was obtained prior to enrollment. This occurred either antenatally or postnatally (before or after the infant reached study eligibility criteria). Reasons for enrollment failure of eligible infants were tracked. This study was approved by the Institutional Review Board at each participating center.

After the correction of hypothermia, anemia, hypoglycemia, and any mechanical cause of low BP, infants were eligible if they had at least two protocol-defined low mean arterial blood pressure (MAP) values (Table I) in the first 24 hours which each met the following criteria: 1) obtained from a UAC with a good wave pattern; 2) not related to medication administration, handling of the infant, technical error, or tracheal suction; 3 ) lasted at least 60 seconds and; 4) low MAP values occurred at least 15 minutes, but no more than six hours, apart. Protocol-defined low BP was derived from previous studies and consensus opinion among the NRN centers to reflect the rise in BP noted with advancing postnatal age and differences in $\mathrm{BP}$ values which occur with invasive versus oscillographic measurements. ${ }^{1-3,8,12-14,29}$ Prior to study entry, perceived low BP or poor perfusion could be managed at the discretion of the clinical care team with only a fluid bolus of $\_0 \mathrm{ml} / \mathrm{kg}$.

This was a double-blinded pilot RCT (ClinicalTrials.gov\#NCT00874393). Enrolled infants were randomized to receive a study infusion (dopamine or placebo) and a study syringe medication (hydrocortisone or placebo) so that they were allocated to one of four groups: 1) dopamine/placebo; 2) dopamine/hydrocortisone; 3 ) placebo/placebo; 4) placebo/ hydrocortisone. This $2 \times 2$ factorial design was chosen because dopamine and hydrocortisone are the most common inotrope and glucocorticoid used for low BP in this population, ${ }^{8}$ a significant drug-drug interaction between the two medications was unlikely, and the effect of the two medications used in combination on infant outcomes was clinically relevant. A placebo/placebo study arm was included because these medications are potentially harmful, not all infants with low BP receive antihypotensive therapy, and there is uncertainty about the clinical efficacy of these medications in this patient population. $4,6,9,12-24$

The study infusion was begun at $6 \mathrm{mcg} / \mathrm{kg} / \mathrm{min}$ of dopamine (or placebo volume equivalent) and increased as often as every 20 minutes to a maximum rate of $15 \mathrm{mcg} / \mathrm{kg} / \mathrm{min}$. Specific $\mathrm{BP}$ criteria for weaning and escalating the study infusion were implemented. The study infusion was stopped when specific BP criteria were achieved or the infant reached the $96^{\text {th }}$ postnatal hour. Seven doses of the study syringe medication were given: an initial dose of 1 $\mathrm{mg} / \mathrm{kg}$ hydrocortisone (or placebo volume equivalent) followed by six doses of $0.5 \mathrm{mg} / \mathrm{kg}$ every twelve hours. If pre-specified study exit criteria were met, open-label antihypotensive therapy was permitted and the clinical care team was notified as to whether or not the infant received dopamine and/or hydrocortisone. The primary outcome was the successful enrollment of 60 infants within one year with a protocol deviation rate of $\_0 \%$. This sample size was chosen to approximate the rate of enrollment for an appropriately powered RCT at all NRN centers over three years and the pilot study was conducted at a subset of network centers to conserve resources while assessing feasibility. Participating centers had experience in conducting RCTs, a principal investigator with publications investigating preterm infant hemodynamics or antihypotensive therapies, and a neonatal group committed to making every effort to complete the study. Protocol deviations included discontinuation of study medications or the use of open-label antihypotensive therapies outside of study protocol, and failure to administer the assigned study medications. Enrolled infants were randomized from a pre-specified sequence allocated by center and administered by an 
investigational pharmacist. Only the investigational pharmacist was aware of assigned medications.

\section{Results}

From December 3, 2009 - December 3, 2010, there were 366 inborn infants 23 - 26 weeks GA admitted to the NICU of a participating NRN center (Figure). Fifty-eight (16\%) infants were eligible for the study and ten (3\%) were enrolled. There were $339(93 \%)$ infants born to mothers who received at least one dose of a glucocorticoid prior to delivery, a UAC was placed in 307 (84\%) infants, and 119 (33\%) infants had protocol-defined low BP. Due to low recruitment, mid-way through the study a seventh center was added and the inclusion criteria were adjusted to allow for study entry without a UAC.

Antenatal consent was sought from 39 women and obtained for four infants born at $23-26$ weeks GA and postnatal informed consent was obtained prior to eligibility for 17 infants. None of these infants met study eligibility criteria. The parents of 20 of the 58 (34\%) eligible infants were not approached for consent. For $13(65 \%)$ of these infants, the attending neonatologist was unwilling to consider enrollment in an RCT due to the infant's critical condition or because s/he felt the infant was deteriorating too quickly to wait for consent. In seven (35\%) cases, one or both parents were not available to provide consent or the mother was unable to consent due to medications affecting her cognition. Of the 38 eligible infants whose parents were approached for consent, $10(26 \%)$ granted consent and their infant was enrolled, $23(61 \%)$ parents refused consent, three (8\%) parents could not make a timely decision regarding consent, and in two (5\%) cases the neonatologist chose to provide open-label therapy for low BP while research personnel were attempting to obtain consent.

The outcomes of the ten enrolled infants are presented in Table II. None of the infants received open-label antihypotensive therapy in the first postnatal week. Although the two infant deaths occurred in the placebo/placebo group, the time of death was distant from enrollment (day 20 for patient six and day 62 for patient seven). Two protocol deviations occurred when the study syringe medication was stopped without meeting exit criteria. The reason for study withdrawal cited in both cases was the risk of intestinal perforation with the possible simultaneous administration of hydrocortisone and indomethacin (administered to facilitate closure of the ductus arteriosus). Of the 48 eligible infants not enrolled in the study, $41(85 \%)$ received at least one therapy for low BP, $34(71 \%)$ received $\geq 20 \mathrm{ml} / \mathrm{kg}$ in fluid boluses, 31 (65\%) dopamine, $11(23 \%)$ hydrocortisone, eight (17\%) dobutamine, and one (2\%) epinephrine.

\section{Discussion}

In this pilot study to assess the feasibility of an RCT of BP management in extremely preterm infants, a low percentage of infants met all eligibility criteria, few eligible infants were enrolled, and a protocol deviation occurred in $20 \%$ of enrolled infants. The study did not achieve its primary objective. Several major factors hindered success, including fewer than anticipated infants with low BP, high rates of early indomethacin administration, difficulty obtaining informed consent, and a lack of physician equipoise. Previous studies suggested approximately half of the infants in this population have low BP, ${ }^{1-7,12-14,16,25}$ however, only one-third of screened infants had protocol-defined low BP. It is unlikely this was due to a different definition of low BP as the criteria used for this study were similar to those of previous reports. ${ }^{4,8,12-14}$ The high rate of antenatal glucocorticoid administration in this study population (93\%) may have improved cardiovascular transition because these medications have been shown to decrease the incidence of low BP in preterm infants. ${ }^{30}$ 
The most common exclusion criterion in this study was prior administration of indomethacin (44\% of screened infants). An unanticipated change in practice at two participating centers early in the study led to the routine, but not universal, use of prophylactic indomethacin for IVH prevention in all infants born 28 weeks GA. In addition, two infants were withdrawn from the study when indomethacin was begun due to concern that the possible concomitant use of hydrocortisone and indomethacin significantly increases the risk of gastrointestinal perforation. ${ }^{22,23}$ Therefore, despite its common use in practice, ${ }^{6,9}$ it may be difficult to include hydrocortisone in an RCT of early BP management.

Difficulties with the consent process resulted in parental consent being successfully obtained for only $17 \%$ of eligible infants. Obstacles included the inefficiency of obtaining antenatal consent, the brief window for obtaining postnatal consent, and family stress surrounding extremely preterm infant birth. Antenatal consent was sought from 39 women and successfully obtained for four infants born within the study GA range, but none of these infants met all eligibility criteria. This is consistent with a previous RCT within the NRN in which antenatal consent was required because infants were enrolled in the delivery room at birth. ${ }^{28}$ In that study, over five women were approached for every infant enrolled. Extrapolating those data to the current study suggests over 35 women would need to be approached prior to delivery for every infant enrolled in an RCT. For 17 infants, postnatal consent was obtained before the infant had low BP. None of these infants met study eligibility criteria, demonstrating the considerable investment of time and effort required to consent parents before entry criteria are met. However, once criteria are met, the window for obtaining consent is short, and several factors may preclude doing so quickly. The birth of an extremely preterm infant is always stressful for parents, and the mother may be receiving medications which interfere with informed consent. In addition, most centers required consent from both parents, but there were instances when paternity was not certain or the father was not immediately available. These factors make obtaining postnatal informed consent so difficult that an RCT of early BP management may not be possible without a waiver of consent as is permitted in the conduct of research of emergency conditions under the United States Food and Drug Administration Code of Federal Regulations \#21CFR50.24. Specific criteria which must be met for this waiver include but are not limited to: study subjects are in a life threatening situation; study participation may directly benefit subjects; investigation could not practicably be carried out without a waiver; an Institutional Review Board approved informed consent process is used when feasible. Although few studies have been conducted on newborn preterm infants using such a waiver, this approach may allow for enrollment of a broader cohort of infants with less risk of a selection bias. ${ }^{28}$ Furthermore, a waiver of consent leaves the option for a deferred information sharing process at which time parents may choose to have their infant opt-out of the study. This seems ethically justifiable given that both treatment and non-intervention are common clinical approaches to this problem. ${ }^{4,6,712-14}$

Physician equipoise is another potential barrier to adequate enrollment without selection bias: $22 \%$ of eligible infants were not approached at physician request, and two infants were given open-label anti-hypotensive therapy while research personnel were attempting to obtain consent. Furthermore, $85 \%$ of eligible infants not enrolled in the study received antihypotensive therapy. Although it may be inappropriate to withhold such treatments from infants with clinical or biochemical evidence of poor perfusion, studies suggest this occurs in only a small subset of infants with low BP. ${ }^{12-14}$ The wide variation in BP management for this population ${ }^{7,16}$ exists in part because there are insufficient data to support a single approach. In the absence of evidence-based guidelines, clinicians make decisions based on previous experience, training, and personal interpretation of the literature, resulting in significant and variable bias. This may hinder the success of an RCT of BP management in 
this population such that a strong commitment to adhere to study protocol should be secured from a large group of neonatologists prior to study initiation.

\section{Acknowledgments}

We are indebted to our medical and nursing colleagues and the infants and their parents who agreed to take part in this study.

\section{Abbreviations}

$\begin{array}{ll}\text { BP } & \text { blood pressure } \\ \text { GA } & \text { gestational age } \\ \text { RCT } & \text { randomized placebo controlled trial } \\ \text { NICU } & \text { neonatal intensive care unit } \\ \text { NRN } & \text { Neonatal Research Network } \\ \text { MAP } & \text { mean arterial systemic blood pressure } \\ \text { UAC } & \text { umbilical arterial catheter }\end{array}$

\section{References}

1. Cordero L, Timan C, Waters H, Sachs L. Mean arterial pressures during the first 24 hours of life in 500-gram birth weight infants. J Perinatol. 2002; 22:348-353. [PubMed: 12082467]

2. Cunningham S, Symon A, Elton R, Changqing Z, McIntosh N. Intra-arterial blood pressure reference ranges, death and morbidity in very low birthweight infants during the first seven days of life. Early Hum Dev. 1999; 56:151-165. [PubMed: 10636594]

3. Lee J, Rafadurai V, Tan K. Blood pressure standards for very low birthweight infants during the first day of life. Arch Dis Child. 1999; 81:F168-F170.

4. Logan J, O'Shea T, Allred E, Laughon M, Bose C, Dammann O, et al. Early postnatal hypotension and developmental delay at 24 months of age among extremely low gestational age newborns. Arch Dis Child Fetal Neonatal Ed. 2011; 96:F321-F328. [PubMed: 21138828]

5. Al-Aweel I, Pursley D, Rubin L, Shah B, Weisberger S, Richardson D. Variations in prevalence of hypotension, hypertension and vasopressor use in NICUs. J Perinatol. 2001; 21:272-278. [PubMed: 11536018]

6. Dempsey E, Barrington K. Diagnostic criteria and therapeutic interventions for the hypotensive very low birth weight infant. J Perinatol. 2006; 26:677-681. [PubMed: 16929346]

7. Laughon M, Bose C, Allred E, O'Shea T, Van Marter LJ, Bednarek F, et al. Factors associated with treatment for hypotension in extremely low gestational age newborns during the first postnatal week. Pediatrics. 2007; 119:273-280. [PubMed: 17272616]

8. Joint Working Party of British Association of Perinatal Medicine and the Research Unit of the Royal College of Physicians. Development of audit measures and guidelines for good practice in the management of neonatal respiratory distress syndrome. Arch Dis Child. 1992; 67:1221-1227. [PubMed: 1444567]

9. Finer N, Powers R, Ou C, Durand D, Wirtschafter D, Gould JB, et al. Prospective evaluation of postnatal steroid administration: a 1-year experience from the California Perinatal Quality Care Collaborative. Pediatrics. 2006; 117:704-713. [PubMed: 16510650]

10. Lundstrom K, Pryds O, Greisen G. The haemodynamic effects of dopamine and volume expansion in sick preterm infants. Early Hum Dev. 2000; 57:157-163. [PubMed: 10735462]

11. Ng P, Lee C, Bnur F, Chan I, Lee A, Wong E. A double-blind randomized, control study of a 'stress dose' of hydrocortisone for rescue treatment of refractory hypotension in preterm infants. Pediatrics. 2006; 117:367-375. [PubMed: 16452355] 
12. Batton B, Batton D, Riggs T. Blood pressure during the first 7 days in premature infants born at postmenstrual age 23 to 25 weeks. Am J Perinatol. 2007; 24:107-115. [PubMed: 17304424]

13. Dempsey E, Al Hazzani F, Barrington K. Permissive hypotension in the extremely low birth weight infant with signs of good perfusion. Arch Dis Child Fetal Neonatal Ed. 2009; 94:F241F244. [PubMed: 19174413]

14. Batton B, Zhu X, Fanaroff J, Kirchner H, Berlin S, Wilson-Costello D, Walsh M. Blood pressure, anti-hypotensive therapy, and neurodevelopment in extremely preterm infants. J Peds. 2009; 154:351-357.

15. Logan J, O'Shea T, Allred E, Laughon M, Bose C, Damman O, et al. Early postnatal hypotension is not associated with indicators of white matter damage or cerebral palsy in extremely low gestational age newborns. J Perinatal. 2011; 31:524-534.

16. Dempsey E, Barrington K. Treating hypotension in the preterm infant: when and with what: a critical and systematic review. J Perinatol. 2007; 27:469-478. [PubMed: 17653217]

17. Ewer A, Tyler W, Francis A, Drinkall D, Gardosi J. Excess volume expansion and neonatal death in preterm infants born at 27 - 28 weeks gestation. Paediatr Perinat Epidemiol. 2003; 17:180-186. [PubMed: 12675785]

18. Greenough A, Cheeseman P, Kavvadia V, Dimitriou G, Morton M. Colloid infusion in the perinatal period and abnormal neurodevelopmental outcome in very low birth weight infants. Eur J Pediatr. 2002; 161:319-323. [PubMed: 12029450]

19. de Zegher F, Van den Berghe G, Devlieger H, Eggermont E, Veldhuis J. Dopamine inhibits growth hormone and prolactin secretion in the human newborn. Pediatr Res. 1993; 34:642-645. [PubMed: 8284103]

20. Filippi L, Pezzati M, Poggi C, Rossi S, Cecchi A, Santoro C. Dopamine versus dobutamine in very low birthweight infants: endocrine effects. Arch Dis Child Neonatal Ed. 2007; 92:367-371.

21. Synnes A, Chien L, Peliowski A, Baboolal R, Lee S, the Canadian NICU Network. Variations in intraventricular hemorrhage incidence rates among Canadian neonatal intensive care units. $\mathrm{J}$ Pediatr. 2001; 138:525-531. [PubMed: 11295716]

22. Peltoniemi O, Kari A, Heinonen K, Saarela T, Nikolajev K, Andersson S, et al. Pretreatment cortisol values may predict responses to hydrocortisone administration for the prevention of bronchopulmonary dysplasia in high-risk infants. J Pediatr. 2005; 146:632-63. [PubMed: 15870666]

23. Watterberg K, Gerdes J, Cole C, Aucott S, Thilo E, Mammel M, et al. Prophylaxis of early adrenal insufficiency to prevent bronchopulmonary dysplasia: a multicenter trial. Pediatrics. 2004; 114:1649-1657. [PubMed: 15574629]

24. Hall R, Kronsberg S, Barton B, Kaiser J, Anand K. Morphine, hypotension, and adverse outcomes among preterm neonates: who's to blame? Secondary results from the NEOPAIN trial. Pediatrics. 2005; 115:1351-1359. [PubMed: 15867047]

25. Fanaroff J, Wilson-Costello D, Newman N, Montpetite M, Fanaroff A. Symptomatic hypotension is associated with neonatal morbidity and hearing loss in extremely low birth weight infants. Pediatrics. 2006; 117:1131-1135. [PubMed: 16585307]

26. Goldstein R, Thompson R, Oehler J, Brazy J. Influence of acidosis, hypoxaemia, and hypotension on neurodevelopmental outcome in very low birth weight infants. Pediatrics. 1995; 95:238-243. [PubMed: 7530835]

27. Martens S, Rijken M, Stoelhorst G, van Zwieten P, Zwinderman A, Wit J, et al. Is hypotension a major risk factor for neurological morbidity at term age in very preterm infants? Early Hum Dev. 2003; 75:79-89. [PubMed: 14652161]

28. Rich W, Auten K, Gantz M, Hale E, Hensman A, Newman N, et al. Antenatal consent in the SUPPORT trial: challenges, costs and representative enrollment. Pediatrics. 2010; 128:e215-e221. [PubMed: 20587676]

29. Short B, VanMeurs K, Evans J. Summary proceedings from the cardiology group on cardiovascular instability in preterm infants. Pediatrics. 2006; 117:S34-S39. [PubMed: 16777820]

30. Been J, Kornelisse R, Rours I, Lima Passos V, De Krijger R, Zimmermann L. Early postnatal blood pressure in preterm infants: effects of chorioamnionitis and timing of antenatal steroids. Pediatr Res. 2009; 66:571-576. [PubMed: 19668111] 


\section{Appendix}

The following investigators, in addition to those listed as authors, participated in this study:

NRN Steering Committee Chair: Michael S. Caplan, MD, University of Chicago, Pritzker School of Medicine.

Case Western Reserve University, Rainbow Babies \& Children's Hospital (U10 HD21364, M01 RR80) - Avroy A. Fanaroff, MD; Bonnie S. Siner, RN; Deanne E. Wilson-Costello, MD.

Duke University School of Medicine, University Hospital, Alamance Regional Medical Center, and Durham Regional Hospital (U10 HD40492, M01 RR30) - Ronald N. Goldberg, MD; C. Michael Cotten, MD MHS; Kimberley A. Fisher, PhD FNP-BC IBCLC; Sandy Grimes, RN BSN.

Eunice Kennedy Shriver National Institute of Child Health and Human Development Stephanie Wilson Archer, MA.

RTI International (U10 HD36790) - Dennis Wallace, PhD; Jeanette O'Donnell Auman, BS; Margaret Cunningham, BS; Carolyn M. Petrie Huitema, MS; James W. Pickett II, BS; Kristin M. Zaterka-Baxter, RN BSN.

Stanford University, Lucile Packard Children's Hospital (U10 HD27880, M01 RR70) David K. Stevenson, MD; M. Bethany Ball, BS CCRC; Melinda S. Proud, RCP.

University of Alabama at Birmingham Health System and Children's Hospital of Alabama (U10 HD34216, M01 RR32) - Namasivayam Ambalavanan, MD; Monica V. Collins, RN BSN MaEd; Shirley S. Cosby, RN BSN.

University of Iowa, Children's Hospital (U10 HD53109, UL1 RR24979, M01 RR59) Edward F. Bell, MD; John A. Widness, MD; Jonathan M. Klein, MD; Karen J. Johnson, RN BSN; Nancy J. Krutzfield, RN MA.

University of New Mexico Health Sciences Center (U10 HD53089, M01 RR997) - Robin K. Ohls, MD; Conra Backstrom Lacy, RN.

The University of North Carolina at Chapel Hill (UL1 RR25747) - Carl L. Bose, MD; Gennie Bose, RN; Cindy Clark, RN.

University of Utah, University Hospital, Intermountain Medical Center, and Primary Children's Medical Center (U10 HD53124, M01 RR64, UL1 RR25764) - Karen A. Osborne, RN BSN CCRC; Jill Burnett, RNC; Cynthia Spencer, RNC; Kimberlee WeaverLewis, RN BSN; Karie Bird, RN; Karen Zanetti, RN; Laura Cole, RN. 


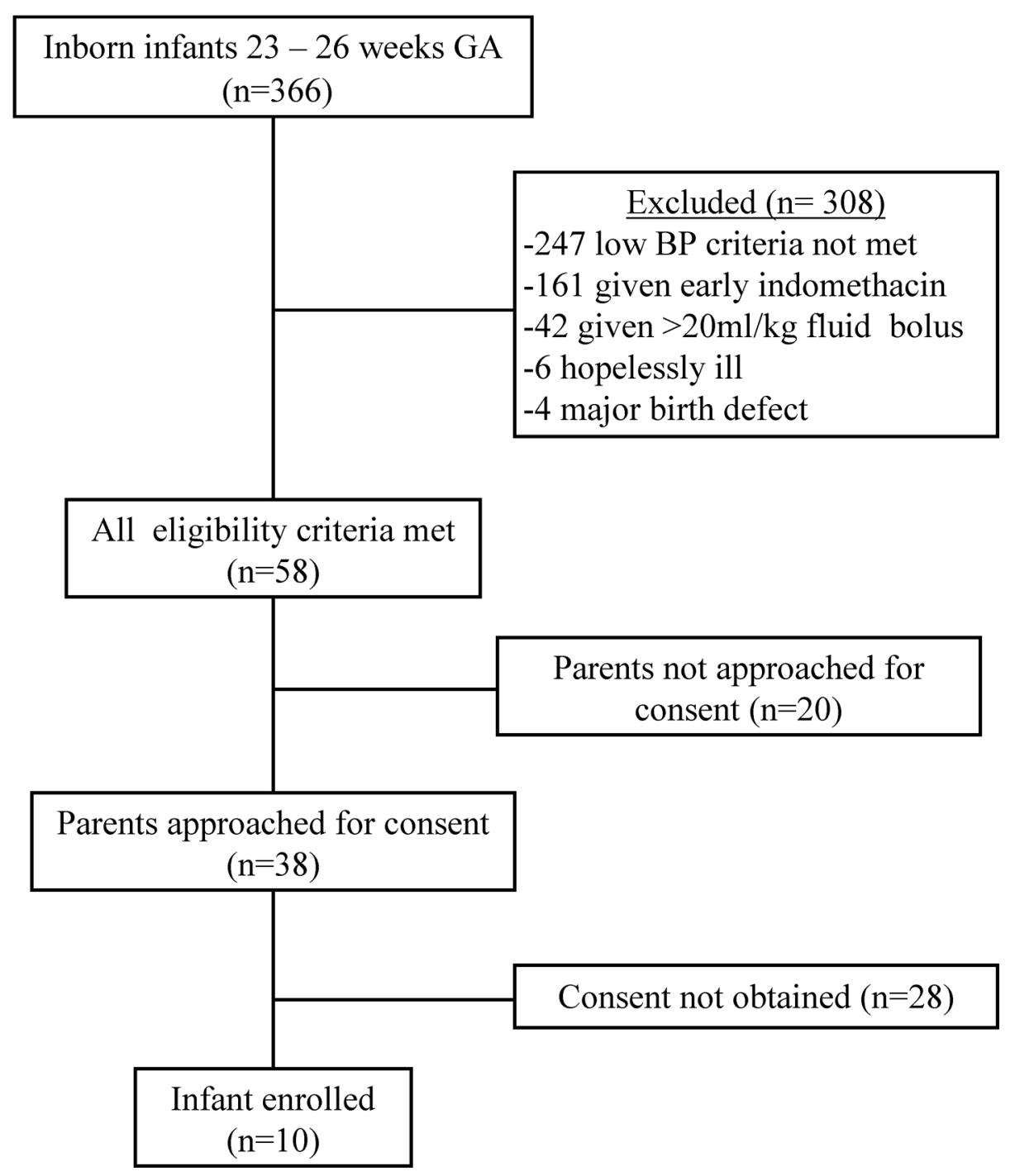

Figure.

Flow diagram of infants. $\mathrm{GA}=$ gestational age; $\mathrm{BP}=$ blood pressure 


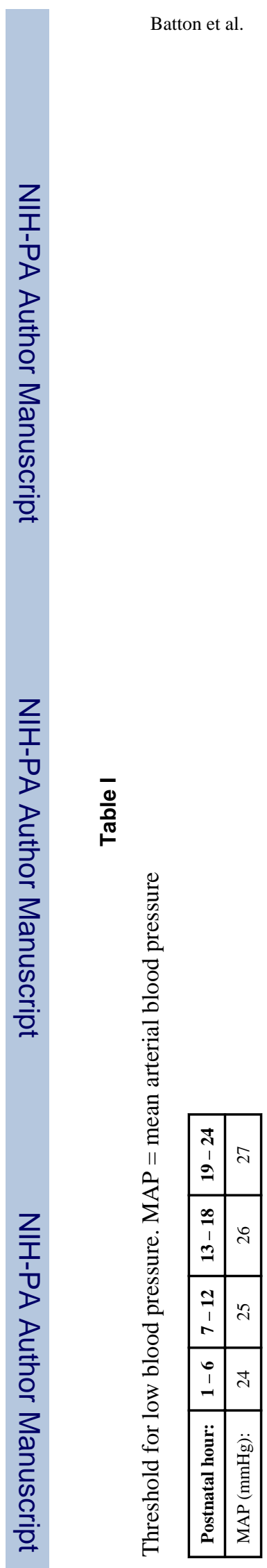

Page 10

J Pediatr. Author manuscript; available in PMC 2013 July 01. 


\begin{tabular}{|c|c|c|}
\hline & 䛼 & $\mathrm{z} z \mathrm{z} z \mathrm{z} z \mathrm{z} z \mathrm{z} z$ \\
\hline$\stackrel{\infty}{\Xi}$ & 商 & $\succ z z \succ z \quad \succ \succ \succ$ \\
\hline II & 2 & $\mathrm{z} z \mathrm{z} z \mathrm{z} z>\mathrm{z} z$ \\
\hline 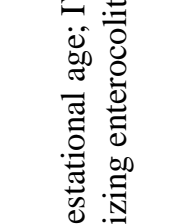 & 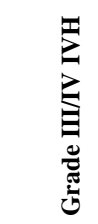 & $z z z z z \succ \succ z z z$ \\
\hline 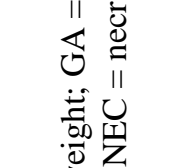 & 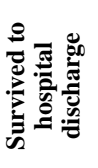 & $\lambda \succ \lambda \succ \succ z \succ \lambda \succ$ \\
\hline 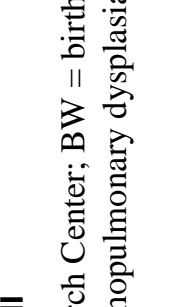 & 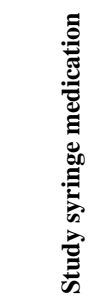 & $\begin{array}{lllll} & & & \\
0 & 0 & 0 \\
0\end{array}$ \\
\hline 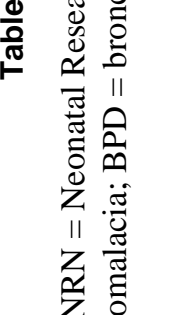 & 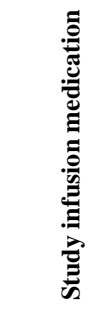 & 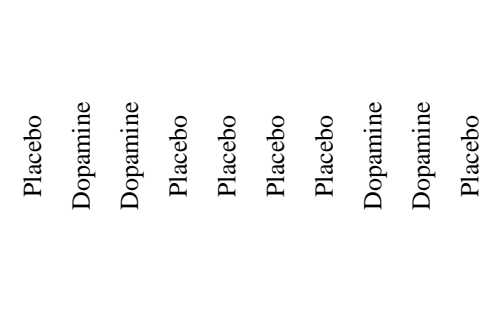 \\
\hline$\underset{\vec{z}}{\stackrel{\vec{z}}{\bar{E}}}$ & Uूँ & 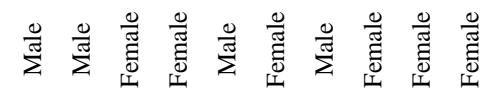 \\
\hline 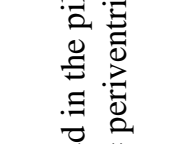 & 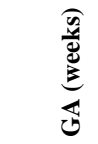 & 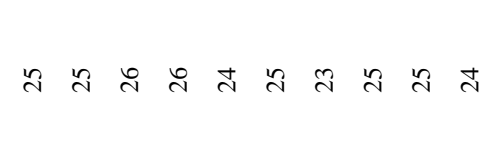 \\
\hline 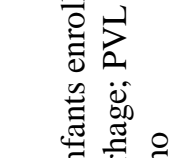 & 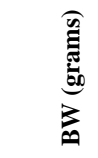 & 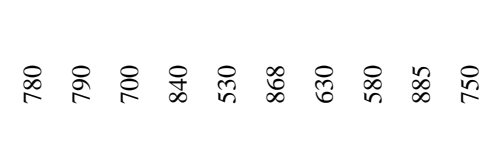 \\
\hline 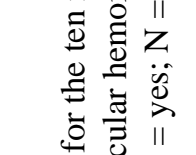 & 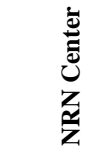 & $\varangle \varangle \varangle \varangle \varangle \infty U \rho \rho \rho$ \\
\hline 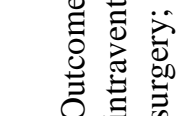 & 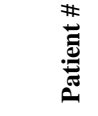 & $-\alpha m+n b r \infty a$ 的 \\
\hline
\end{tabular}

J Pediatr. Author manuscript; available in PMC 2013 July 01. 